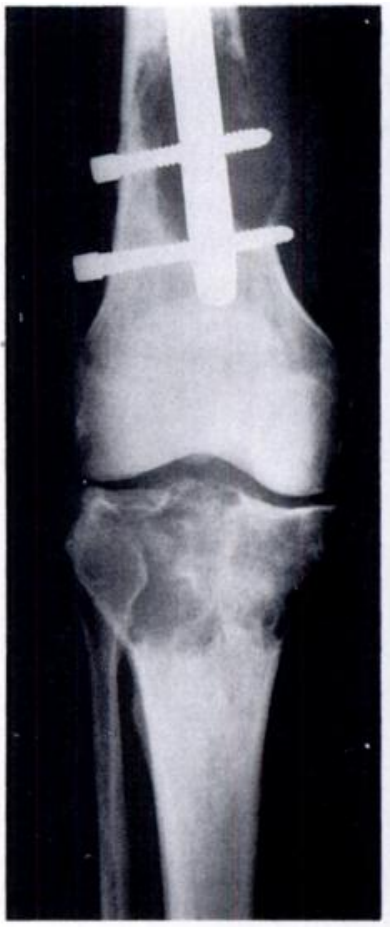

Fig. 1

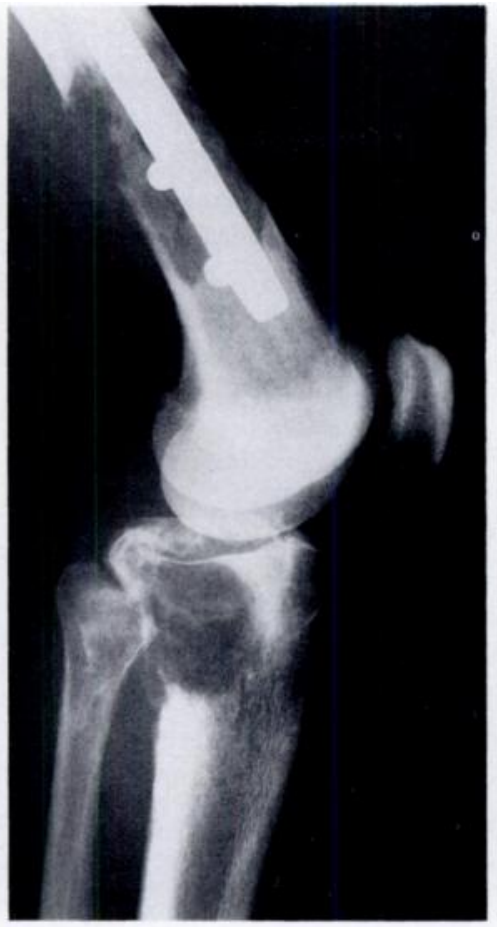

Fig. 2

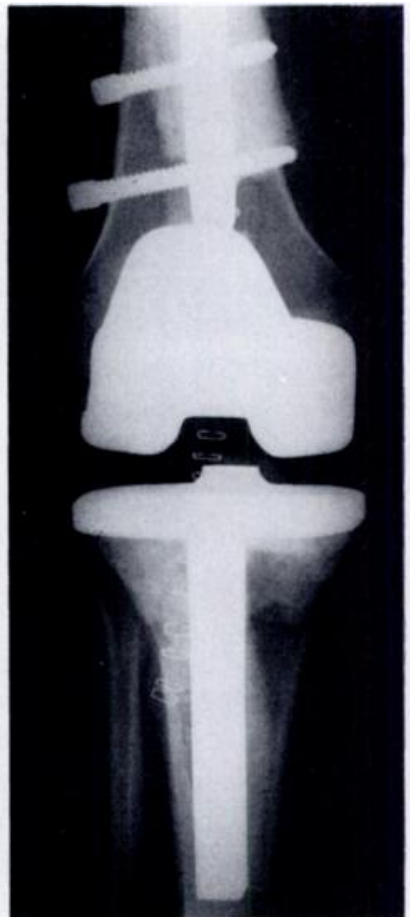

Fig. 3

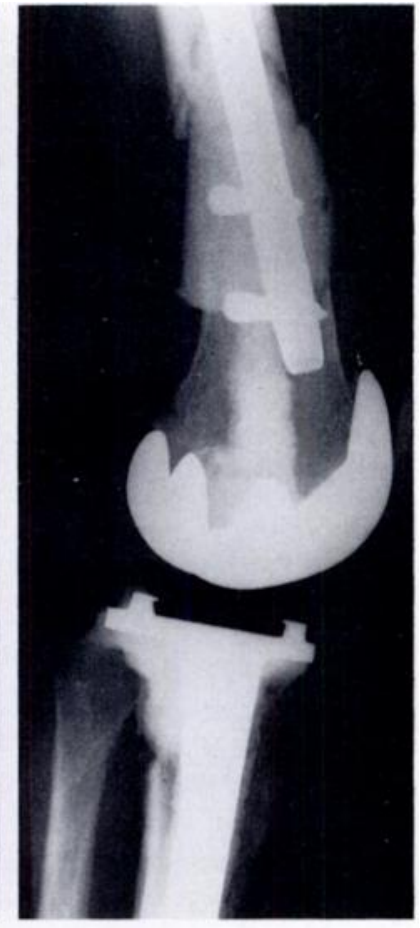

Fig. 4

the insertion of a collateral ligament or the patellar tendon has been destroyed and extensor function may be lost. A custom-made device is often necessary, and the delay involved may render this impractical in patients with a short life expectancy.

Operative treatment of skeletal metastases may have a high morbidity and must be aimed at improving the patient's quality of life. Our case illustrates that where survival even of a few months is likely, total knee arthroplasty can be an extremely rewarding and successful procedure.

No benefits in any form have been received or will be received from a commercial party related directly or indirectly to the subject of this article.

\section{REFERENCES}

Beauchamp CP, Sim FH. Lesions of the tibia. In : Sim FH, ed. Diagnosis and management of metastic bone disease. New York: Raven Press, $1988: 207-12$.

Fidler M. Incidence of fracture through metastases in long bones. Acta Orthop Scand 1981; 52:623-7.

Galasko CSB. Skeletal metastases. London: Butterworths, 1986:134-5.

Harrington KD. Orthopedic management of metastatic bone disease St Louis: CV Mosby, 1988.

\title{
AUTOLOGOUS BLOOD TRANSFUSION IN A DISTRICT HOSPITAL
}

\author{
A. GOEL, A. N. EDWARDS, N. C. WEST
}

An alternative to homologous blood transfusion is the use of the patient's own blood (autotransfusion) also known as autologous or predeposited blood transfusion (Kay 1987; Lee and Napier 1990). We have been using autotransfusion for over two years for hip and knee replacements; a brief review is presented.

A. Goel, FRCS, MS (Orth), Orthopaedic Registrar

A. N. Edwards, FRCS, Orthopaedic Consultant

N. C. West, MRCP, MRCP Path, Consultant Haematologist

West Cumberland Hospital, Whitehaven, Cumbria CA28 8JG, England.

Correspondence to Mr A. Goel.

(C) 1991 British Editorial Society of Bone and Joint Surgery $0301-620 X / 91 / 3 R 12 \$ 2.00$

J Bone Joint Surg [ Br] 1991; 73-B :517-8.
Methods. Our current protocol is that when the patient is put on the waiting list, he is given a fact sheet about autologous transfusion and the procedure is explained; if willing, a note is made in the records. Two weeks before the operation is due to take place the patient is admitted as a day case and reassessed from the point of view of surgery and autologous transfusion. If a decision is made to go ahead with the operation and if the patient is fit, a sample of blood is sent for haemoglobin estimation and blood screening.

The patient then goes to the haematology department where the results of the tests are recorded and, if the haemoglobin is adequate, one unit of blood is taken. He returns to the orthopaedic wards and after a brief period of observation is sent home with a prescription 
for haematinics. The patient returns the next week as an out-patient to the haematology department and a further unit of blood is taken. A week later the patient is admitted to the orthopaedic wards for surgery.

Results. During the two years from January 1988 to December 1989, 96 patients were willing to undergo autologous transfusion; of these, six had too low a haemoglobin. The number of units of blood collected was 166 ; of these, 36 were discarded but the remainder were used.

Sixteen patients $(17.8 \%)$ required banked blood as well as their own; five patients needed no blood at all. In all, $57.3 \%$ of the patients who were undergoing joint replacement donated blood.

The only complication recorded was the wastage of six units of blood as a result of air entering the pack. A serious possible complication is mistransfusion of blood due to errors in labelling. Although we did not experience this complication it is recommended that strict procedures should be followed for collection, storage and records; the responsibilities of everyone taking part must be well defined.
Conclusions. From our experience with autologous transfusion we believe that it is a safe and effective means of meeting the transfusion requirements of patients undergoing elective orthopaedic procedures. It eliminates the risk of blood-borne infections such as AIDS and hepatitis and it promotes patient involvement in the surgical procedure. It can be used with other techniques, such as blood salvage and planned haemodilution to minimise blood transfusion. Autologous blood transfusion is a simple and inexpensive process which does not require expensive equipment. It can be successfully used in any hospital.

We would like to thank Mr W. Musgrave, Senior MLSO at the West Cumberland Hospital and the rest of the haematology department for their co-operation.

No benefits in any form have been received or will be received from a commercial party related directly or indirectly to the subject of this article.

\section{REFERENCES}

Kay LA. The need for autologous blood transfusion. Br Med J 1987; 294:137-9.

Lee D, Napier JAF. Autologous transfusion. Br Med J 1990; 300: $737-40$.

\title{
LESSONS IN THE INVESTIGATION OF IRRITABLE HIP: FAILURE OF ULTRASOUND TO DETECT HAEMARTHROSIS
}

\author{
A. G. MATHIE, M. K. D’A. BENSON, D. J. WILSON
}

Ultrasonography has proved a sensitive indicator of effusion in a child's hip, based on the detection of anterior displacement of the iliofemoral ligament (Wilson, Green and MacLarnon 1984). The degree of this displacement usually relates to the size of effusion, but under certain circumstances this correlation breaks down, rendering ultrasonographic assessment unreliable.

Case reports. Two children with previously undiagnosed haemophilia each presented with an irritable hip. The first, a healthy eight-year-old boy, developed progressive pain and restricted movement of his right hip over the six days prior to his referral. The second, a 14-month-old boy, was admitted with a 48-hour history of reluctance to bear weight on his left leg. There was no history of trauma or of systemic upset in either child, and on examination both children were apyrexial. The affected hips were held fixed in flexion, abduction, and external

A. G. Mathie, MA, MB BS

M. K. D'A. Benson, FRCS, Consultant Orthopaedic Surgeon

D. J. Wilson, MRCP, FRCR, Consultant Radiologist

Nuffield Orthopaedic Centre, Windmill Road, Headington, Oxford OX3 7LD, England.

Correspondence to Mr M. K. D'A. Benson.

(C) 1991 British Editorial Society of Bone and Joint Surgery 0301-620X/91/4R23 \$2.00

J Bone Joint Surg [Br] 1991 ; 73-B:518-9. rotation. Plain radiographs clearly demonstrated hip stand-off which, in the 14-month-old boy, was considerable and amounted to frank subluxation (Fig. 1a). Ultrasound examinations failed to detect significant

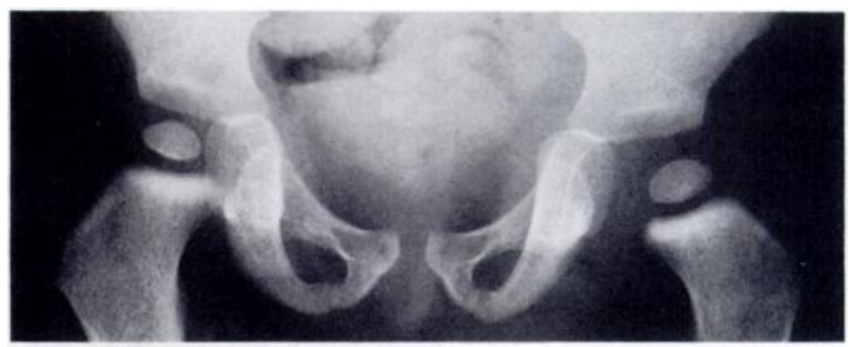

Fig. 1a

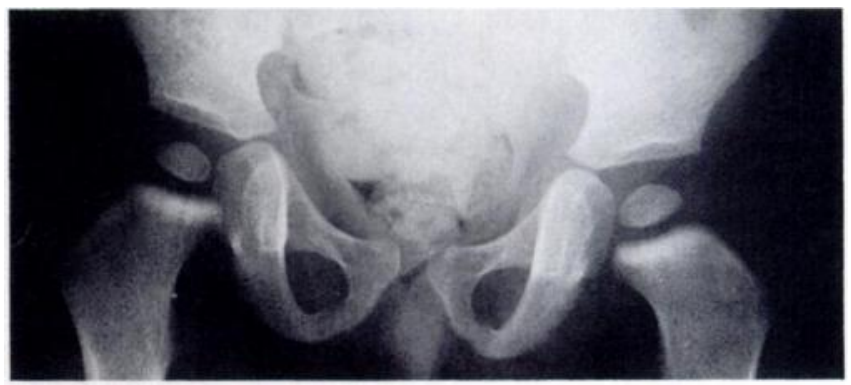

Fig. $1 b$

Figure la showing left hip subluxation; lb showing reduction after aspiration of $5 \mathrm{ml}$ of blood. 